\title{
Research on the Electrical Automation Control System Reliability Enhancement Improvement Ways from the System Feedback Angle
}

\author{
Guojuan Zhou \\ Beijing Institute Of Economics And \\ Management,Beijing,100102 \\ Shuli Meng \\ Beijing Institute Of Economics And \\ Management,Beijing,100102
}

\author{
Rensheng Wei \\ Beijing Institute Of Economics And \\ Management,Beijing,100102 \\ Hong Chen \\ Beijing Institute Of Economics And
Management,Beijing, 100102
}

\begin{abstract}
In this paper, we conduct research on the electrical automation control system reliability enhancement improvement ways from the system feedback angle. Electronic control system for the development of the national economy has an important role in promoting, on the national life and the enterprise production has important influence. Effective application in general electric power system automation control technology can further improve the basic efficiency of the operation of the power system, to bring economic benefits to the electric power enterprise, should be in strict accordance with the technology to improve the reliability of the system, reasonable choice of the automation system operating mode, to take measures to prevent wrong operation of the control equipment, effectively improve the working efficiency of the system. Therefore, our research propose the innovative ways of solution that is meaningful and has special significance.
\end{abstract}

Keywords- System Feedback, Control System, Reliability Enhancement, Electrical Automation.

\section{Introduction}

Electrical automation is an important symbol of industrial modernization and the core of the modern advanced science and technology, is to make the operation of the product, control and monitoring can in no one directly involved in the case, according to a predetermined plan or program automatically to technology. Electrical automation control system of the characteristics, functions and design concept could be summarized as the follows. (1) Compared with the heat engine equipment, electric control system of controlled objects, small volume, less operating frequency is low, but with the advantages of rapid and accurate. Due to the protection of the electrical equipment to demand higher automatic equipment has high reliability and rapid response ability and anti-interference ability. (2) Based on the characteristics of the electrical control, electrical automation control system to realize the generator transformer set of effective control of the electrical system, etc. request to the microprocessor control system to design software, to meet the current degree of the automation control, to ensure that the controller can release the bad industrial environment. (3) Electrical automation control systems are mainly centralized monitoring and the remote monitoring, field bus monitoring three design methods. The characteristics of centralized monitoring way are by the processor centralized system function, advantage is simple in design, protection requirements is low, operation maintenance convenience.

Power system automation software application is the main project, in the operation of the software applications, often can appear many times back to correct situation, need operator to set the access, to prevent the illegal operation. Monitoring software should have a certain tolerance at the same time, to prevent the 
occurrence of general operating personnel work mistakes, that functional impact on the system. And be able to automatically restore blank phenomena caused by accidental failures. Stand accused of operators through the change is the monitoring host action command and realize remote control distance and on-site operation works is a kind of more, multistage automation control mode.

It is the main purpose of the present electrical automation distributed control. Its itself is a kind of intelligent equipment and automatic system connection, that will these branches branch of the serial communication bus which holds the listed developmental trend. (1) For the PC controller with high reliability and simple operation and maintenance, a growing number of primary manufacturers in the production process of different levels and PC control scheme, trying to shorten product development and production cycle and improve the quality of the products. (2) In as large as possible within the scope of the electrical automation system structure of the control system in general is very important. Management can also use the Internet to the production process, production equipment management and supervision. (3) On the basis of professional knowledge as the guide, a deeper understanding of the operating personnel to install, repair the operating system. Safety production technology and basic operation and professionals is the trend for the future development of electrical automation in our country, enterprises in the process of the production can't ignore these fundamental problems. In this paper we conduct theoretical research on electrical automation control system reliability enhancement improvement ways from the system feedback angle [1-3].

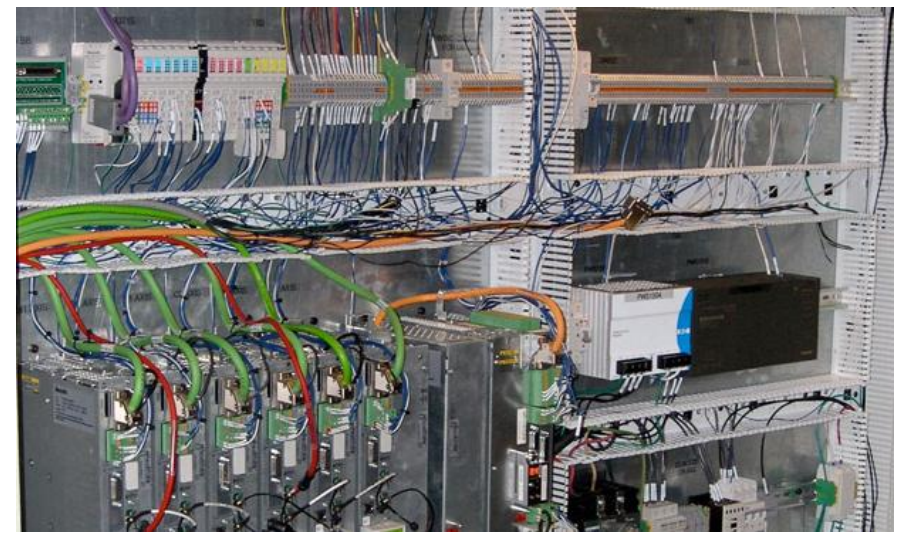

Figure 1. The Demonstration of Electrical Automation Control System

\section{Our Proposed Methodology}

The Principles of Electrical Control. Control by the simplification to the intelligent development of electrical control technology, manual shift towards automation, operation manual operation to the informationization progress. As the core position of the electrical control circuit is the foundation of promoting continuous development. Enterprise to improve its efficiency and market competitiveness, must promote the electrical control circuit design and the combination of science and technology, as promoting the development of the scientific efficiency [4].

The three principles that will influence the efficiency of the control for system could be organized as follows. (1) Transmission delay. Time needed for packet transmission in the actual transmission media, its size depends on the size of the packet, the network bandwidth and transmission distance. (2) Packet queuing delay. When the network is busy or basic packet 
collision occurs, waiting for the network free of waiting time. (3) Delay information. The sender sent information encapsulated into the packets and into the queue time required.

Design of the electrical control circuit analysis and design method of the two most commonly used logic method. The first method analysis method is also called the basic experience method, it to the designer's own experience, etc. The demand is higher. It is primarily the designer according to the requirements of mechanical equipment and the actual process, the comprehensive some typical links and then combined with the experience of the designer design the actual need of control route scheme. The second method is more often used in the design of the complex circuit. It starting from the actual requirements of production process, according to the operation characteristics and the working state of the machine table, find the main electrical appliances and components of the logical relationship, the two as the independent variable and the dependent variable respectively, using the logic algebraic expression, combines with the truth table, comprehensive analysis of its design. First of all, designers need to clear the master all the start and stop the signal components, and ensure that each operation element working condition and requirements, puts it into a complete work process, to grasp the signal of each element. Second, designers determine its logical correspondence, is accused of components in electrical control only coil electricity and loss, contact the closing and opening of the two states. Will start and stop signals as variables, and its change rule and logic rule is matched. Through this method relatively simple logical expressions are obtained. Finally, according to the operation good logical expression designs the circuit diagram. Generally simple design scheme suitable for analysis, design of complex empirical method can also be used, but the empirical method for designers in the design of the complex circuit while the rich practical experience is required.

The System Feedback Angle. Power system under periodic load perturbation produce oscillation, in the case of a disturbance is not loses stability. But when the disturbance to a certain extent, the system will appear chaotic oscillation, which is a non-periodic, seems to be no rules, sudden or basic paroxysmal pathological electromechanical oscillation, in severe cases can lead to losing the stability reconciliation that could be expressed as the formula one [5-6].

$$
\frac{d}{d t} w(t)=-\alpha \sin \delta(t)-\gamma w(t)+\rho+F \cos \beta t
$$

(1)

System caused by the periodic load disturbance chaos oscillation control, the basic idea is to use the single variable linear feedback force system is chaotic or unstable state to a stable state. So, this method is simple and easy to implement. The general nonlinear excitation controller is based on state feedback design, with the rotor angle back to the reference for the generator control target. When the angle of the rotor of the generator is held constant, the voltage of the machine is associated with the running conditions of general system, including the network parameters, such as mechanical power generators. After the disturbance of power system network parameters and operation mode change, while the controller generator rotor angular displacement can be to disturbance in front of the rotor angle, but is likely to cause the voltage of the machine from its reference value. 


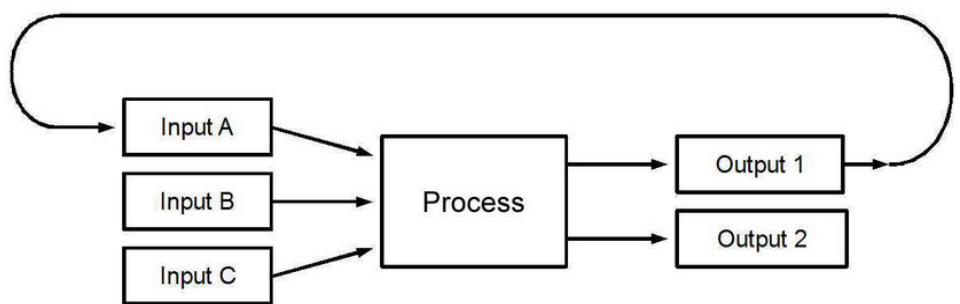

Figure 2. The Demonstration of the System Feedback

Correspondingly, the design pattern could be generally summarized as the follows. (1) Feedback control method has the advantage that: no need to use any other than the system output or core state variables controlled system information such as the change of the system parameters had no effect on small parameter change on the system, thus can make the dynamic behavior of the original system is maintained. (2) Chaotic motion with initial value sensitivity and general development trends of long unpredictability, it allows the use of small disturbance of the control system feedback track this is a chaotic system. Therefore, it can make people don't have to avoid chaos, but likely to chaos control and utilization for the further optimization of the systematic performance.

The System Reliability. The factors that affect the reliability of the control system design is more, including electromagnetic disturbance in the process of application, the power interference and signal lines introduce the general interference factors. At present, we are the most common is the radiation electromagnetic interference, the interference will produce a certain degree of damage to equipment, especially when the power is in the high power electric equipment line impedance coupling to form the power interference. In addition, the control system itself also has some problems, such as its own errors caused by signal lines to introduce interference, grounding system chaos caused by interference and the problems existing in the actuator control system factors [7].

Reliability index under the condition of the known and reliability criteria given, according to the assessment criteria to evaluate the main wiring system reliability measure of the ability to meet the demand of electric power system analysis on the reliability of the substation main wiring can be used as the basis for the design and evaluation of the main electrical wiring, and can choose the optimal solution and the best way to run as a basis, is found through the analysis of the main electrical wiring weak links, in order to arrange plans to overhaul. The testing methodologies could be generally listed as the follows. (1) To simplify the design. It is on the premise of guarantee the product performance requirements as simplify product design as much as possible. To simplify the design can improve the inherent reliability and the basic reliability of general products. (2) Redundant design. Is the system or equipment for high reliability, high safety and high viability of one of the design methods especially when the components or components quality and reliability level is low, the general design has been unable to meet the requirements of the equipment reliability as redundancy technology has important application value. (3) Derating design. It is to make the working stress of the components or general equipment to work under appropriate amount value of the below components or equipment, so as to reduce the failure rate while improving the reliability of the using purpose. 


\section{System/Mission Reliability}

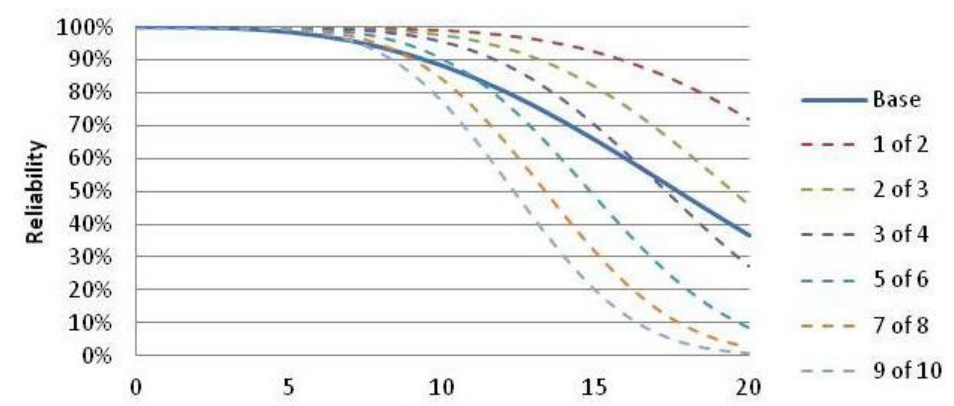

Figure 3. The Demonstration of the System Reliability Development Pattern

\section{The Automation Control and} Enhancement Methods. Electrical automation in machinery and the electrical appliances, power electronics, automatic control, computer technology, and the other modern technology, its main characteristic is strong and weak electricity mechanical and the electrical combination, combination of software and hardware, and electrical automation technology provides more convenient for production and life, have application universality and professionalism, with high efficiency, the broad application prospect. As the electrical automation control equipment reliability under the attention of more and more high, the equipment reliability testing is becoming more and more important. In the process of the actual detection, we select the appropriate detection method based on real condition, lest affect test results for basic electrical automation control equipment in the actual operation of facing a series of problems [8].

In mechanical and electrical integration products, electrical automation control system has a very important role, which is equivalent to the human brain, used for information processing and control. So, in the design of the basic control system of electrical automation must follow the corresponding process. In accordance with the requirements of control to finalize the design scheme of the electrical automation control system, and then the control algorithm, and select the appropriate microcomputer, work out the overall design of the control system of electrical automation content, finally carried out the design of the software and the hardware. Although electrical automation control system design process is relatively complex, but must from set out actually, when the design consideration of a core centralized monitoring, field general line monitoring and remote monitoring, only then to be able to match the requirements of control system is set up [9].

Under this guidance, we summarize the further application and optimization scenarios as the list. (1) The application of smart technology. Intelligent control technology is to use electrical automation technology in power system is more complex system of intelligent management and the real-time monitoring, so as to improve the sensitivity of the power system and deal with the problem timely. (2) The application of simulation technology. Automation not only can the application of the simulation technology for power system running in the massive data analysis and processing, but also through the way of data model for experimental personnel provide the virtual experiment environment and the system operation environment, can be in the running fault in power system with simulation analysis to improve the accuracy of diagnosis and troubleshooting. (3) The application of multiple integration technology. Multiple integration technology is a for all of the control system for unified management in electric power system technology, the application of this technology makes integration with the integration of the various management department at the same time, 
and can use a number of the other advanced technology for systematic modification.

\section{Conclusion}

In this paper, we conduct research on the electrical automation control system reliability enhancement improvement ways from the system feedback angle. Electrical automation control technology to promote the modernization process of the chemical production. Due to the complexity of chemical production process often irregular, it is necessary to establish effective mathematical model of the system. Advanced control technology applied in chemical production, system identification and the dynamic mathematical model is set up, determine the relationship between the input and output variables, such as, dealing with chemical field noise data, variable calculation, etc. In addition, the application of field control technology has promoted the electrical automation reform of the chemical production, has the very good development prospect. Under this background, we propose the system reliability enhancement improvement ways from system feedback angle to serve as the new paradigm for the further design of the corresponding systems.

\section{References}

[1] Wang, Gang Quan. "Research on Artificial Intelligence Technology of Electrical Automation Control." Applied Mechanics and Materials. Vol. 624. Trans Tech Publications, 2014.

[2] Hashimoto, Tomoaki. "Computational Simulations on Stability of Model Predictive Control for Linear Discrete-time Stochastic Systems." International Journal of Computer, Electrical, Automation, Control and
Information Engineering 9 (2015): 1385-1390.

[3] Gray, Alex D., et al. "Optimising the use of electrical energy within the waste water industry through improved utilization of process control and automation." Power Engineering Conference (UPEC), 2015 50th International Universities. IEEE, 2015.

[4] Lehmann, Beat, et al. "Intermediate complexity model for model predictive control of integrated room automation." Energy and Buildings 58 (2013): 250-262.

[5] Wang, Bowen. "The Application and Study of MATLAB in Electrical Engineering and Its Automation." International Conference on Advances in Mechanical Engineering and Industrial Informatics. Atlantis Press, 2015.

[6] Peng, Chen, Min-Rui Fei, and Engang Tian. "Networked control for a class of T-S fuzzy systems with stochastic sensor faults." Fuzzy Sets and Systems 212 (2013): 62-77.

[7] WEI, Jie, et al. "Research of Development and Control Strategy on Magnetic Suspension Wind Turbine." Electrical Automation 1 (2013): 004.

[8] Xu, Changbo, Wei Lu, and Chunwen Li. "A control strategy for multi-modular shunt active power filters applied to high-power systems." Dianli Xitong Zidonghua(Automation of Electric Power Systems) 37.8 (2013): 117-122.

[9] Benedetti, Miriam, et al. "Energy consumption control automation using Artificial Neural Networks and adaptive algorithms: Proposal of a new methodology and case study." Applied Energy 165 (2016): 60-71. 